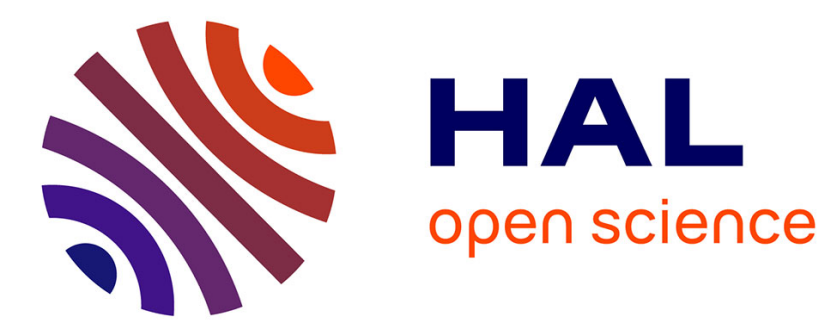

\title{
TOWARDS A FRAMEWORK OF INTER-OBSERVER ANALYSIS FOR MULTIMEDIA QUALITY ASSESSMENT
}

\author{
Ulrich Engelke, Yohann Pitrey, Patrick Le Callet
}

\section{- To cite this version:}

Ulrich Engelke, Yohann Pitrey, Patrick Le Callet. TOWARDS A FRAMEWORK OF INTEROBSERVER ANALYSIS FOR MULTIMEDIA QUALITY ASSESSMENT. Third International Workshop on Quality of Multimedia Experience (QoMEX), Sep 2011, Mechelen, Belgium. pp. 183- 188, 10.1109/QoMEX.2011.6065700 . hal-00608314

\section{HAL Id: hal-00608314 https://hal.science/hal-00608314}

Submitted on 12 Jul 2011

HAL is a multi-disciplinary open access archive for the deposit and dissemination of scientific research documents, whether they are published or not. The documents may come from teaching and research institutions in France or abroad, or from public or private research centers.
L'archive ouverte pluridisciplinaire HAL, est destinée au dépôt et à la diffusion de documents scientifiques de niveau recherche, publiés ou non, émanant des établissements d'enseignement et de recherche français ou étrangers, des laboratoires publics ou privés. 


\title{
TOWARDS A FRAMEWORK OF INTER-OBSERVER ANALYSIS IN MULTIMEDIA QUALITY ASSESSMENT
}

\author{
Ulrich Engelke, Yohann Pitrey, Patrick Le Callet \\ IRCCyN UMR no 6597 CNRS, Ecole Polytechnique de l'Universite de Nantes, \\ rue Christian Pauc, La Chantrerie, 44306 Nantes, France
}

\begin{abstract}
In multimedia quality assessment, observer ratings are typically averaged into mean opinion scores (MOS) to obtain a subjective ground truth for a set of stimuli. Valuable information about individual observer rating behaviour and interobserver differences is lost during this process. Such information, however, can be useful to improve subjective experiment procedures and quality of experience prediction models. In this paper, we therefore present an inter-observer analysis framework that tackles the quality assessment problem from an inverse angle, setting the focus on individual observer differences rather than stimuli differences. The framework consists of a set of procedures for inter-observer analysis as well as the necessary considerations during pre-processing and post-processing. The aim of this paper is to raise awareness that sole consideration of MOS simplifies quality assessment too much, especially given the ever increasing complexity of multimedia quality assessment.
\end{abstract}

Index Terms - Inter-observer differences, multimedia quality assessment, inter-rater coefficients, data mining.

\section{INTRODUCTION}

Quality of Experience (QoE) has been of major concern for multimedia service providers in recent years to complement more traditional paradigms such as Quality of Service (QoS) [1]. The objective is a transition from an application-centered to an user-centered delivery of multimedia services with the main goal being the satisfaction of the users' experience. This task has been considerably complicated through recent advances in coding and networking technology that facilitated ubiquitous usage of a broad range of multimedia applications on a variety of digital devices, ranging from mobile phones over laptop computers to high definition television in the home. For multimedia service providers it is crucial to automatically measure the QoE provided to satisfy the consumers' expectations. Hence, a considerable research effort has been devoted to the development of models that predict human quality perception. However, despite the increased complexity of multimedia quality assessment, subjective experiment outcomes are still reduced to an average vote over all participants, the

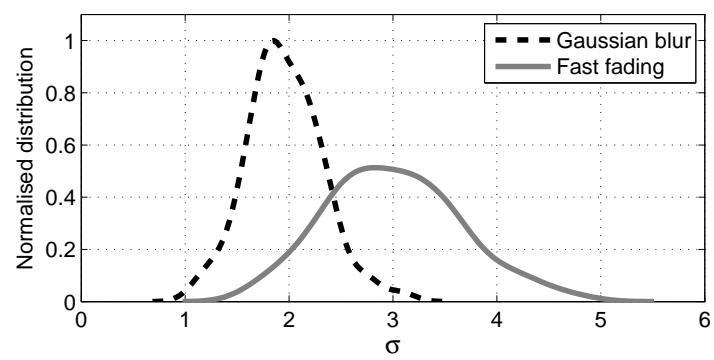

Fig. 1. Normalised distributions of standard deviations $\sigma$ for images distorted through Gaussian blur or fast fasting [2].

mean opinion score (MOS). The agreement between participants is measured using confidence intervals $(\mathrm{CI})$ and related quantities, such as the standard error of the mean.

The use of MOS and CI may have sufficed in the context of 'simple' assessment tasks, such as in the evaluation of content with single, additive distortions. However, contemporary multimedia applications constitute a considerably more complex scenario, as a multitude of factors impact on the quality, including, source coding, error-prone networks, error concealment, re-scaling, and display devices. With such an increased complexity of the assessment task, the disagreement between observers may increase. This is illustrated in Fig. 1. Here, normalised distributions of standard deviations over all observers are shown for all Gaussian blur and fast fading distorted images in the LIVE image quality database [2]. It can be seen, that generally the observer agreement is considerably higher for the Gaussian blur distorted images, as indicated by the lower standard deviations. This can be attributed to the comparably simpler assessment task as the fast fading distorted images exhibit more complex distortion patterns. Not only system related factors, however, but also other internal (subjective) and external (environmental) factors have been found to contribute to observer disagreement. In [3], for instance, it was shown that the confidence of human observers into their quality ratings is inversely related to the CI. Different internal and external factors have been profiled in [4] in the context of audiovisual 3D perception, revealing individual quality preferences with respect to certain modalities.

To address the shift to user-centric multimedia services, it 


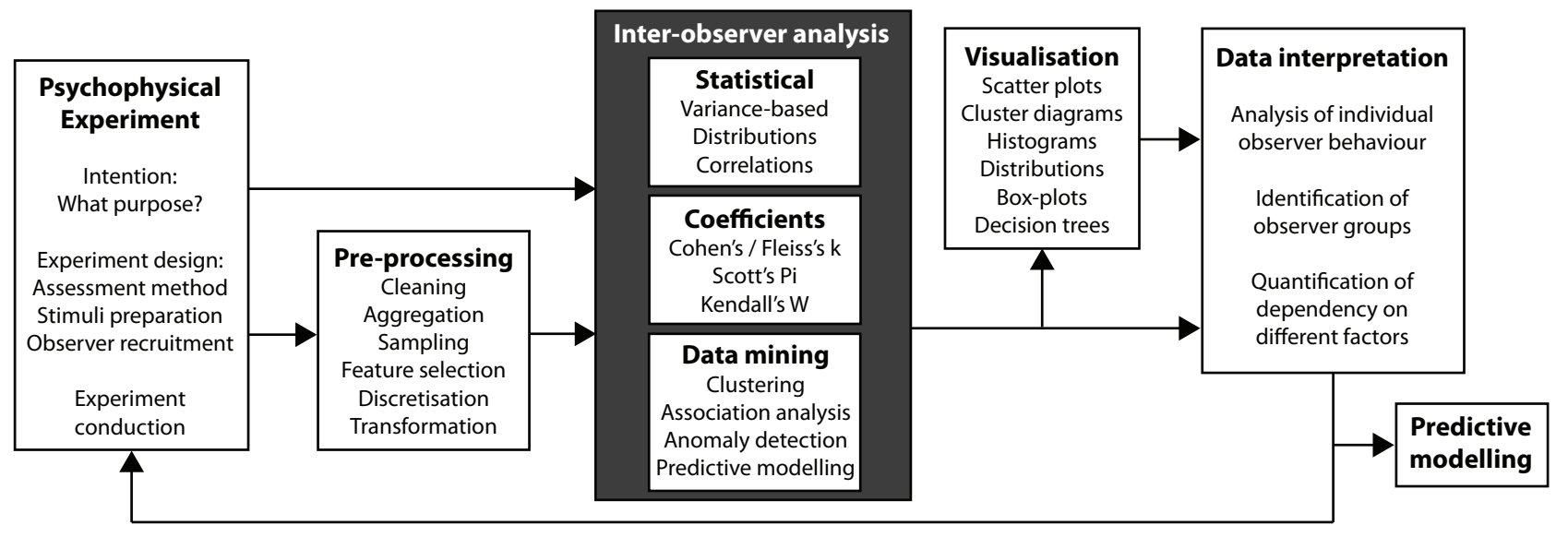

Fig. 2. Framework for inter-observer analysis in multimedia quality assessment.

is crucial to go beyond considerations of simple MOS and to gain a better understanding of individual observer perception in the context of multimedia services. The importance of evaluating human observer differences has already been recognised in other research disciplines, especially in medical analysis [5]. Not just the disagreement between observers as such needs to be quantified (as is done with CI) but more insight into rating behaviour needs to be obtained by analysing individual observers and by determining possible observer groups that exhibit similar behaviour. To identify whether differences are due to internal factors (e.g. personal preferences) or external factors (such as environmental influences or artifacts in the experimental design) is one goal of inter-observer analysis. This in turn is instrumental to improve psychophysical experiment procedures and to design elaborate QoE prediction models that more adequately reflect the complexity of modern multimedia applications. To this end, conventional psychophysical assessment strategies and data analysis methodologies deployed by the multimedia quality assessment community may need to be revisited and additional, unconventional procedures need to be established.

\subsection{Towards a framework of inter-observer analysis}

To address the above issues, we present in this paper a framework for assessment of inter-observer variability. The objectives in this context are twofold. Firstly, we aim to raise awareness that inter-observer differences should not be neglected in multimedia quality assessment. This is particularly true in case of multimodal assessment and for novel applications such as 3D video, where observer differences are expected to be large. Secondly, we propose a set of existing and novel techniques to measure inter-observer variations and apply them in a multimedia quality assessment context.

The integral parts of the framework are presented in Fig. 2 and are discussed in detail in this paper. Generally, the framework can be divided into three parts; preparation, inter-observer analysis, and data exploitation. To take full advantage of the framework, special considerations should already take place during the psychophysical experiment and the pre-processing of the data. These preparatory issues are explained in Section 2. The focus of this paper is on the heart of the framework, the inter-observer analysis. We consider three different means to quantify inter-observer differences; statistics, inter-rater coefficients, and data mining [6], which are discussed in detail in Section 3. The subsequent visualisation and interpretation of the results is essential for a thorough exploitation and comprehension of the inter-observer analysis and is briefly discussed in Section 4, along with some implications on experimental design and predictive modelling. Finally, conclusions are drawn and future directions are given in Section 5.

The ultimate goal of the framework is to answer many important questions, including: What are the underlying causes of observer differences? To what degree do they depend on internal and external factors, as well as the experimental design? Can random variations be distinguished from systematic variations? Are there particular observer groups that can be identified? Can individual observer differences or observer groups be incorporated into predictive quality models to improve their performance? It should be noted that the current state of the framework is by no means considered to be complete. The purpose is rather to initiate re-consideration of conventional quality assessment, discuss representative candidate methodologies for inter-observer assessment, and to pose open questions that need more attention.

\section{PREPARATION}

This section discusses special considerations that should be taken into account in the conduction of the experiment and the data pre-processing to maximise the outcomes of the interobserver analysis. A 3D quality experiment [7] is briefly introduced that is used throughout the paper as a means to better illustrate the different aspects of the framework. 


\subsection{Psychophysical experiments}

Although analysis of inter-observer differences is of great interest for any existing experiment data, one may in fact take it particularly into account already during preparation of future experiments. This applies to all aspects of experiment design and especially to the stimuli preparation, observer recruitment, and the assessment methodology.

The presented stimuli are usually selected with the aim to determine system performance by carefully choosing parameters from a realistic parameter space. Similar considerations can be applied to identify observer differences, where content classes can be carefully chosen such as to identify personal preferences or distortions can be introduced such as to identify thresholds with respect to certain modalities.

The recruitment of observers does not necessarily need to be solely based on the discrimination between experts and non-experts, as is often done. To identify differences in rating behaviour between observers, one may consider further demographic data and observer profiles as a criterion for observer recruitment. For instance, recruitment of observers who have a 3D television and observers who do not may aid in identifying whether there is a difference in the comfort that these observer groups experience when viewing 3D content.

Depending on the application and the intended purpose, data should be recorded during the experiment in addition to the quality ratings. For instance, to identify observer confidence, additional confidence scores and response times have been recorded in [3]. To assess the comfort during 3D video viewing, additional comfort scores were collected in [7]. Such complementary information aids to gain a deeper insight into the rating behaviour and the perception of the presented stimuli. Questionnaires handed out before and after the experiment can further provide valuable information.

In summary, the increasing complexity of quality assessment in contemporary multimedia applications needs to be reflected already during the conduction of the experiment.

\subsection{1. $3 D$ video quality experiment}

The outcomes of a 3D video quality experiment are used here to illustrate the concepts of the framework. Details of the experiment can be found in [7] and are summarised in the following. The experiment was conducted in two laboratories in France and Sweden, however, in this paper we consider only the former experiment. Eleven different 3D video sources (SRC) were used along with 15 hypothetical reference circuits (HRC) to create a total of 165 processed video sequences (PVS). The HRCs consisted of a number of different coding scenarios, including multi view coding and H.264 simulcast, to identify their impact onto perceived quality. The videos were viewed by 22 naive observers. In addition to the quality scores, comfort scores were collected to measure the degree of discomfort due to the $3 \mathrm{D}$ experience. Both scores were rated on a 5-point absolute category rating (ACR) scale.

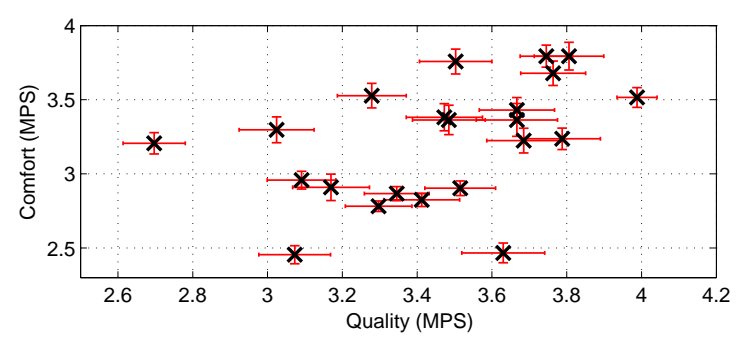

Fig. 3. Quality and comfort MPS including error bars.

\subsection{Pre-processing}

Pre-processing typically involves detection of observers that exhibit a considerably different rating behaviour compared to the majority of the observer population. These outliers are often removed according to certain criteria such as the ones specified by VQEG [8]. To identify the causes of the disagreement rather than directly rejecting these observers is one goal of the inter-observer framework.

Inter-observer analysis based on data mining techniques, as discussed in Section 3.3, needs particularly careful preprocessing to prepare the data with the aim to improve the quality of the outcome [6]. For instance, dimensionality reduction using principal component analysis (PCA) based methods can be used on high dimensional data to improve comprehension of the results. Furthermore, discretisation of continuous scale data is usually needed for classification algorithms. To identify certain patterns it can further aid to transform the data into a more suitable format or representation before deploying the data mining algorithm.

\section{INTER-OBSERVER ANALYSIS}

In this framework we consider three means of inter-observer analysis, statistics, inter-rater coefficients, and data mining techniques, which are explained in the following sections.

\subsection{Statistics}

Simple statistics such as the mean, standard deviation, skewness, and kurtosis are usually computed over the observer population to quantify the characteristics of the ratings for particular PVS or HRC. Inversely, these simple statistics can be utilised to quantify rating behaviour of each observer over the presented stimuli. Figure 3 shows the mean PVS scores (MPS) for of the comfort versus quality scores for all 22 participants. It can be clearly seen that there is a wide range of different average quality and comfort ratings. To identify whether this 'bias' is due to the observers' different perception of the PVS, due to systematic error in the experiment design, or simply by chance is of great importance.

The scatter plot in Fig. 3 gives an overview of the averaged differences amongst the observers, however, it does 

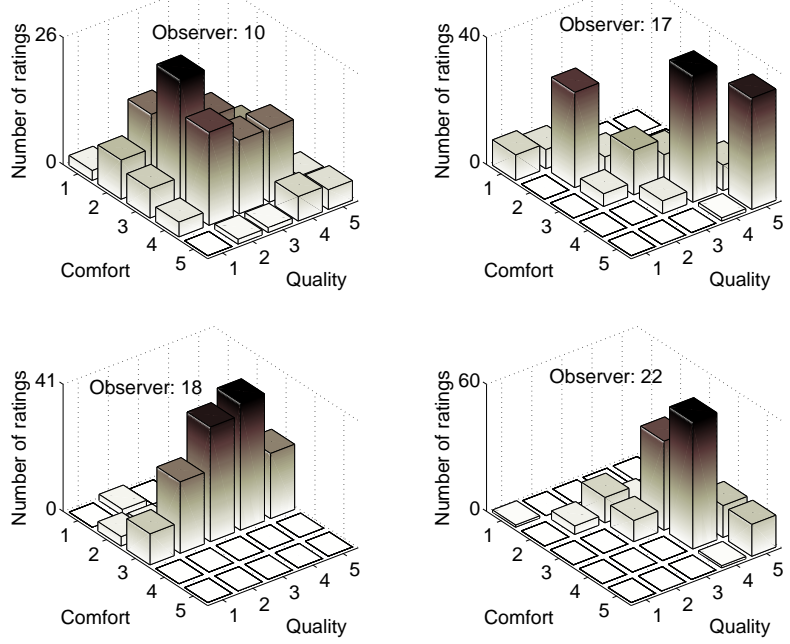

Fig. 4. Co-joint quality and comfort ratings for 4 observers.

not provide detailed information regarding the differences in rating behaviour. These differences are better accounted for in statistics that consider the distribution of the scores, such as the histograms of the co-occurrences of quality and comfort scores illustrated in Fig. 4. Here, one can clearly identify considerable differences in rating behaviour between the four observers. Whereas observer 10 uses both scores in different combinations, observer 17 tends to give the same scores for a particular PVS, meaning, the scores are highly correlated. Observer 18, on the other hand uses a wide range of quality scores but utilises mainly the middle of the comfort scale. Observer 22 focuses mainly on a very narrow range for both scores. These differences in rating behaviour should not be ignored and their underlying cause needs to be investigated.

The difference between any two observers can be quantified using correlations, such as the Pearson linear correlation coefficient illustrated in Fig. 5. The upper figure shows the correlations between all observers for the quality scores (above diagonal) and the comfort scores (below diagonal). The correlations are generally higher for the quality scores, which is supported by the marginal distributions in the lower plot. The lower agreement on comfort can have multiple reasons, including, more difficulty in rating or larger differences in perception, maybe due to the novelty of 3D applications.

In [9] it is argued that correlation is different from agreement and thus, should not be used to measure inter-observer agreement. This argument is supported by the simple example in Fig. 6. Here, the diagonal represents perfect correlation and perfect agreement. The solid dots, however, represent perfect correlation but poor agreement, due to the distance of the dots from the diagonal. The circles, on the other hand, represent high agreement but zero correlation.

Typically, analysis of variance (ANOVA) is used to measure whether or not differences amongst two or more factors

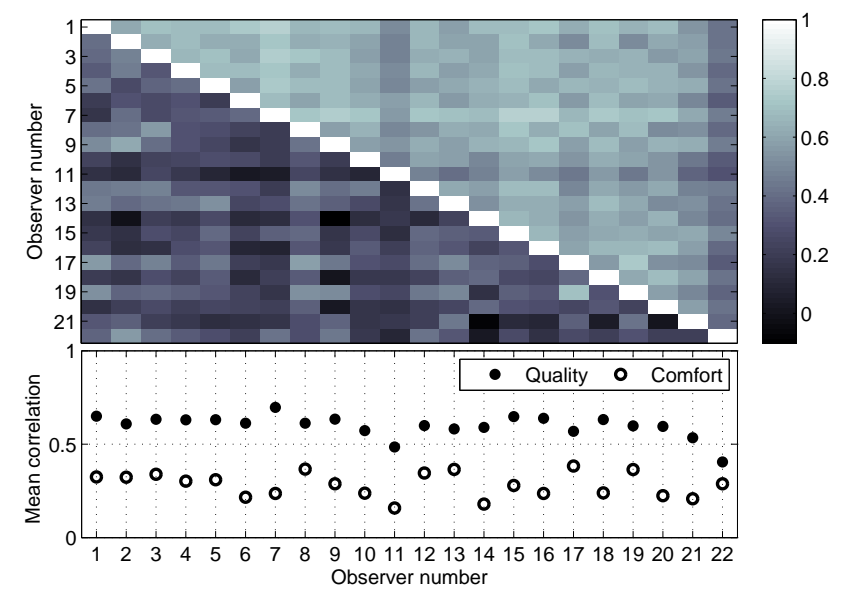

Fig. 5. Inter-observer correlations for quality scores (above diagonal) and comfort scores (below diagonal) and their marginal distributions.

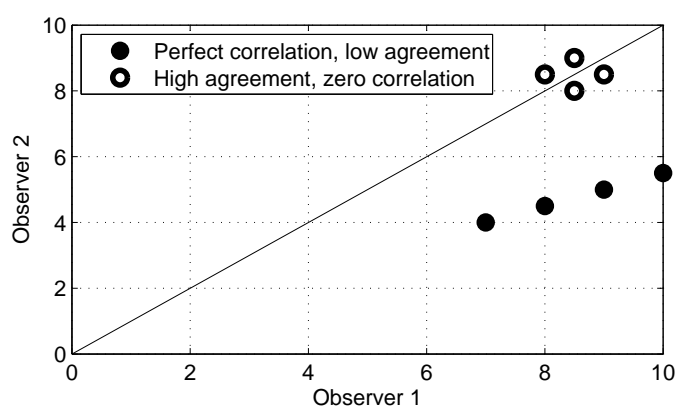

Fig. 6. Difference between correlation and agreement [9].

are significant. However, in [9] it is argued that ANOVA makes two assumptions that not necessarily hold in analysing observer disagreement. Firstly, ANOVA assumes that observers have the same error variance and, secondly, it assumes that correlations between any two observer pairs are the same throughout the population. Especially the latter assumption is particularly questionable in case of subjective experiments with non-expert observers.

Given the above, so called inter-rater coefficients that are widely used in medical research and decision making could constitute a more suitable measure of inter-observer analysis.

\subsection{Inter-rater coefficients}

Inter-rater coefficients take into account that a certain amount of agreement between observers is due to chance alone [5]. As such, they aim to measure the true disagreement by identifying and segregating the chance agreement. Early coefficients include Kendall's $W$ [10] and Scott's Pi [11]. Cohen's $\kappa[12]$, probably the most widely used inter-rater coefficient, is an extension of Scott's Pi which does not assume that ob- 
Table 1. Confusion matrix applied to a 5-point scale.

\begin{tabular}{|c|c|c|c|c|c|c|c|}
\hline & \multicolumn{5}{|c|}{ Ratings observer A } & \multirow[b]{2}{*}{ Total } \\
\hline & & 1 & 2 & 3 & 4 & 5 & \\
\hline \multirow{5}{*}{$\begin{array}{c}\text { Ratings } \\
\text { observer } \\
\text { B }\end{array}$} & 1 & $n_{11}$ & $n_{12}$ & $n_{13}$ & $n_{14}$ & $n_{15}$ & $n_{1}$. \\
\hline & 2 & $n_{21}$ & $n_{22}$ & $n_{23}$ & $n_{24}$ & $n_{25}$ & $n_{2}$ \\
\hline & 3 & $n_{31}$ & $n_{32}$ & $n_{33}$ & $n_{34}$ & $n_{35}$ & $n_{3}$ \\
\hline & 4 & $n_{41}$ & $n_{42}$ & $n_{43}$ & $n_{44}$ & $n_{45}$ & $n_{4}$ \\
\hline & 5 & $n_{51}$ & $n_{52}$ & $n_{53}$ & $n_{54}$ & $n_{55}$ & $n_{5}$ \\
\hline \multicolumn{2}{|c|}{ Total } & $n_{\cdot 1}$ & $n_{\cdot 2}$ & $n_{\cdot 3}$ & $n_{.4}$ & $n_{.5}$ & $n$ \\
\hline
\end{tabular}

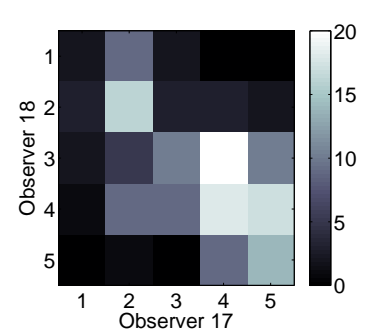

(a)

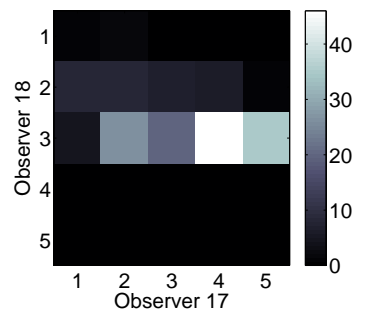

(b)
Fig. 7. Confusion matrices between observers 17 and 18 for (a) quality and (b) comfort scores.

servers have the same rating distributions. Fleiss' $\kappa$ [13] is an extension of Cohen's $\kappa$ from two to multiple observers.

Cohen's $\kappa$ is essentially based on a confusion matrix between two observers, which is given in Table 1 for a 5-point scale. For illustration, the confusion matrices between observers 17 and 18 are given in Fig. 7 for quality and comfort scores. One can see that the agreement between the two observers is higher for the quality scores, as indicated by the high magnitudes around the diagonal.

The $\kappa$ coefficient is computed as

$$
\kappa=\frac{p_{o}-p_{e}}{1-p_{e}}=\frac{\frac{1}{n} \sum_{i=1}^{5} n_{i i}-\frac{1}{n^{2}} \sum_{i=1}^{5} n_{i} \cdot n_{\cdot i}}{1-\frac{1}{n^{2}} \sum_{i=1}^{5} n_{i} \cdot n_{\cdot i}}
$$

with $p_{o}$ and $p_{e}$ denoting the observed and expected agreement, respectively. In [14] it is stated that the conventional $\kappa$ is only suitable for nominal data and is inappropriate for ordinal data, such as given with ordinal scales in quality assessment. The reason being, that in ordinal scales there are different levels of disagreement and, hence, a weighted $\kappa$ is recommended that penalises disagreements in correspondence to their severeness as follows

$$
\kappa_{w}=\frac{\frac{1}{n} \sum_{i=1}^{5} \sum_{j=1}^{5} w_{i j} n_{i j}-\frac{1}{n^{2}} \sum_{i=1}^{5} \sum_{j=1}^{5} w_{i j} n_{i} \cdot n_{\cdot i}}{1-\frac{1}{n^{2}} \sum_{i=1}^{5} \sum_{j=1}^{5} w_{i j} n_{i} \cdot n_{\cdot i}} .
$$

Note that the unweighted $\kappa$ is a special case of the weighted $\kappa$ with $w_{i j}=1$ for $i=j$ and $w_{i j}=0$ for $i \neq j$. Here, we use linear weights $w_{i j}=1-0.25|i-j|$.

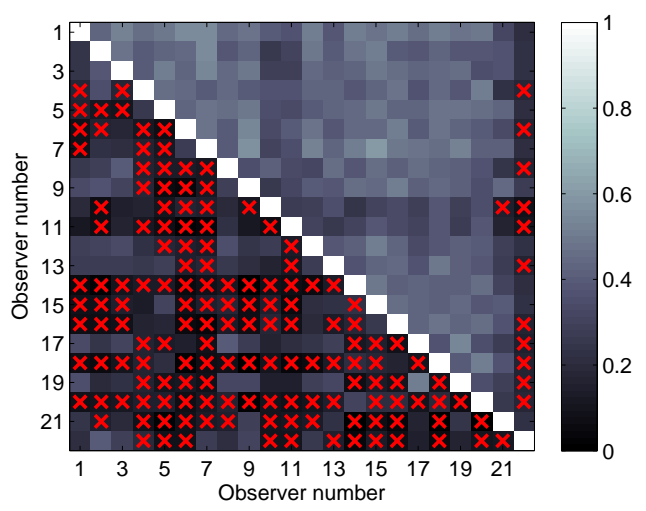

Fig. 8. Cohen's weighted $\kappa_{w}$ between observers for quality scores (above diagonal) and comfort scores (below diagonal).

Both the weighted and unweighted $\kappa$ are measured on a scale from -1 to 1 , with 1 indicating perfect agreement and 0 meaning agreement by chance. Values below zero indicate agreement less than chance and are very rare. To improve interpretation of $\kappa$, an ordinal scale was introduced in [15] as follows: $\leq 0=$ poor, $0.01-0.2=$ slight, $0.21-0.4=$ fair, $0.41-$ $0.6=$ moderate, $0.61-0.8=$ substantial, and $0.81-1=$ almost perfect. However, the authors themselves admitted that the choice of ranges and labels was somewhat arbitrary, for which reason we do not consider them here.

In Fig. 8, Cohen's weighted $\kappa_{w}$ between all observers is presented for the quality scores (above diagonal) and the comfort scores (below diagonal). The red crosses additionally mark the $\kappa_{w}$ where the null hypothesis that agreement is accidental is accepted (based on a $p$ value of 0.05 ). It can be seen that the $\kappa_{w}$ are generally lower and the chance agreement is higher for the comfort scores as compared to the quality scores. It is further apparent, that observer 22 not only exhibits chance agreement with the majority of observers for the comfort scores, but also for the quality scores. This disagreement is also indicated in the correlations (see Fig. 5), however, its severeness is more apparent using the $\kappa_{w}$ coefficient.

\subsection{Data mining}

Within this framework, data mining techniques are instrumental to identify patterns in the experiment data and to determine possible observer groups. These techniques are particularly useful in cases where an extensive amount of data is available and when the dimensionality of the assessment task exceeds easy comprehension of the results. Furthermore, powerful and freely available data mining software, such as the Waikato Environment for Knowledge Analysis (WEKA) [16] or RapidMiner [17], allow for straightforward application of a wide range of data mining algorithms. In [6], data mining techniques are generally separated into four categories, each of which finds their application within inter-observer analysis. 


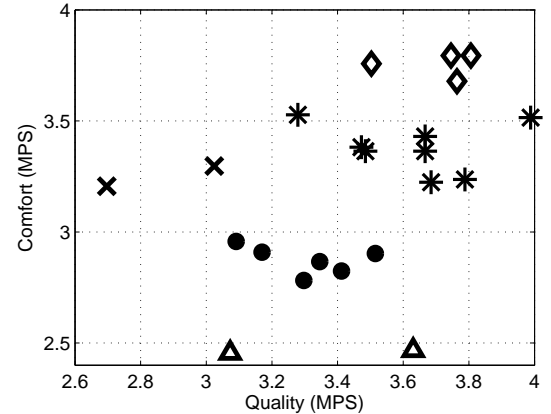

Fig. 9. Clustering using EM and 5 clusters.

Clustering [18] is considered to be particularly useful to identify user groups with respect to one or multiple different attributes. A range of different algorithms [19], such as $k$ means, expectation maximisation (EM), and support vector machines, provide powerful tools to identify these patterns. We applied EM clustering with 5 clusters to the MPS presented in Fig.3. The results are presented in in Fig. 9 with different markers indicating the association of a data point to a cluster. It can be seen, that 5 clusters are created by the EM algorithm, however, the separation between clusters is not always very distinct. Considerations of inter-observer analysis already during the quality experiment (see Section 2.1) may improve cluster separation.

Association analysis can be used to discover strongly associated attributes in the data, which is particularly useful for experiment data where a large number of different factors are considered. Implementations such as the Apriori algorithm facilitate efficient extraction of patterns in such high dimensional experiment data.

Anomaly detection devotes itself to the identification of data that is significantly different from the rest of the data and is thus particularly useful for outlier detection. Various techniques utilising proximity, density, or clustering-based algorithms cover outlier detection for low to high dimensional experiment data.

Predictive modelling is useful to infer models from the experiment data using classification (e.g. decision trees) or regression-based methods for discrete and continuous data, respectively. Typically, predictive modelling is conducted using the MOS, however, more detailed information about individual observer ratings may help to derive models that take into account the complexity of multimedia QoE assessment.

In general, data mining provides powerful tools for interobserver analysis. However, a careful selection of suitable algorithms along with a thorough parameterisation are essential to maximise the usefulness of the output. The pre-processing of the data, as discussed in Section 2.2, is crucial to maximise the performance of the data mining algorithms. Strong integration of data mining techniques into inter-observer analysis is an ultimate objective of the proposed framework.

\section{DATA EXPLOITATION}

As with conventional quality assessment, appropriate visualisation of the analysis outcomes is essential for comprehension of the results with respect to the intended goals of the experiment. In case of inter-observer analysis the focus should thus be on emphasising the differences amongst observers rather than different PVS. An appropriate visualisation is the first step towards successful data interpretation and in fact, in some cases visualisation alone can help to identify patterns in the data (often referred to as visual data mining). In case of high-dimensional data, a technique called online analytical processing (OLAP) [6] is particularly useful as it aggregates data to aid subsequent analysis. Several of these visualisation techniques have been discussed in this paper, a more comprehensive selection is given in [6].

Interpreting the data and making sense of it is possibly the most difficult part of the framework. From the discussion thus far it is obvious that there are indeed strong differences between observers in terms of bias and rating patterns. However, to identify what exactly these differences stem from (internal versus external factors, systematic versus random differences, ...) is a highly difficult task and in fact, the authors believe that data interpretation would benefit from a careful deployment of the entire framework, starting with the experimental design.

Thoroughly interpreted inter-observer analysis outcomes are expected to be beneficial towards the improvement of subjective experiment procedures as well as for the improvement of QoE prediction models. For instance, in [7] it was found that there was a bias between the scores that were given in the two experiments, which could be caused by differences in the conduction of the experiment. However, they could also be due to cultural differences between the French and Swedish participants or other factors that yet need to be identified.

\section{CONCLUSIONS}

We propose a framework of inter-observer variability analysis in the context of multimedia quality assessment. The paper serves to both, raise awareness to the importance of inter-observer analysis and to provide representative tools to conduct it. The different parts of the framework have been discussed in relation to a $3 \mathrm{D}$ video quality experiment. A considerable number of references is provided as a roadmap for the reader to further explore the discussed framework.

The results presented here are only a first step towards a more complete framework and many aspects are yet to be investigated. For instance, other inter-observer coefficients need to be investigated, including bivariate coefficients [20] that simultaneously account for two variables (here: quality and comfort). Also, the range of available data mining techniques is vast and yet to be explored to fully exploit their power in identifying patterns in the data. The authors also 
plan to extend the framework to other domains, such as to the identification in observer differences of gaze patterns recorded through eye tracking [21]. Finally, the framework is planned to be applied to exhaustive data sets (such as the VQEG common set) and a dedicated experiment is planned taking the objective of inter-observer analysis into account already during the design of the procedures.

\section{REFERENCES}

[1] D. Soldani, M. Li and R. Cuny (Ed.), QoS and QoE Management in UMTS Cellular Systems, John Wiley \& Sons, 2006.

[2] H. R. Sheikh, Z. Wang, L. Cormack, and A. C. Bovik, "LIVE image quality assessment database release 2," http://live.ece.utexas.edu/research/quality, 2005.

[3] U. Engelke, A. J. Maeder, and H.-J. Zepernick, "On confidence and response times of human observers in subjective image quality assessment," in Proc. of IEEE Int. Conf. on Multimedia and Expo, June 2009, pp. 910-913.

[4] D. Strohmeier, S. Jumisko-Pyykkö, and U. Reiter, "Profiling experienced quality factors of audiovisual 3D perception," in Proc. of Int. Workshop on Quality of Multimedia Experience, June 2010, pp. 70-75.

[5] M. Banerjee, M. Capozzoli, L. McSweeney, and D. Sinha, "Beyond kappa: A review of interrater agreement measures," The Canadian Journal of Statistics, vol. 27, no. 1, pp. 3-23, Mar. 1999.

[6] P. N. Tan, M. Steinbach, and V. Kumar, Introduction to Data Mining, Addison Wesley, 2006.

[7] K. Wang, M. Barkowsky, R. Cousseau, K. Brunnström, R. Olsson, P. Le Callet, and M. Sjöström, "Subjective evaluation of HDTV stereoscopic videos in IPTV scenarios using absolute category rating," in Proc. of IS\&T/SPIE Stereoscopic Displays and Applications XXII, Jan. 2011, vol. 7863.

[8] Video Quality Experts Group, "Final report from the Video Quality Experts Group on the validation of objective models of multimedia quality assessment, phase I," VQEG, Sept. 2008.

[9] M. Haber and H. X. Barnhart, "Coefficients of agreement for fixed observers," Statistical Methods in Medical Research, vol. 15, no. 3, pp. 255-271, June 2006.

[10] M. G. Kendall and B. Babington Smith, "The problem of m rankings," The Annals of Mathematical Statistics, vol. 10, no. 3, pp. 275-287, Sept. 1939.

[11] W. Scott, "Reliability of content analysis: The case of nominal scale coding," Public Opinion Quarterly, vol. 19, no. 3, pp. 321-325, 1955.

[12] J. Cohen, "A coefficient of agreement for nominal scales," $E d$ ucational and Psychological Measurement, vol. 20, no. 1, pp. 37-46, Apr. 1960.

[13] J. L. Fleiss, "Measuring nominal scale agreement among many raters," Psychological Bulletin, vol. 76, no. 5, pp. 378-382, 1971.

[14] J. Sim and C. C. Wright, "The kappa statistic in reliability studies: Use, interpretation, and sample size requirements," Physical Therapy, vol. 85, no. 3, pp. 257-268, Mar. 2005.

[15] J. R. Landis and G. G. Koch, "The measurement of observer agreement for categorical data," Biometrics, vol. 33, no. 1, pp. 159-174, 1977.

[16] M. Hall, E. Frank, G. Holmes, B. Pfahringer, P. Reutemann, and I. H. Witten, "The WEKA data mining software: An update," SIGKDD Explorations, vol. 11, no. 1, pp. 10-18, June 2009.
[17] Rapid-I GmbH, "RapidMiner," http://rapidi.com/content/view/181/190/, 2011.

[18] P. Berkhin, "A survey of clustering data mining techniques," in Grouping Multidimensional Data, J Kogan, C. Nicholas, and M. Teboulle, Eds., pp. 25-71. Springer Berlin Heidelberg, 2006.

[19] X. Wu, V. Kumar, J. R. Quinlan, J. Ghosh, Q. Yang, H. Motoda, G. J. McLachlan, A. Ng, B. Liu, P. S. Yu, Z. H. Zhou, M. Steinbach, D. J. Hand, and D. Steinberg, "Top 10 algorithms in data mining," Knowledge and Information Systems, vol. 14, no. 1, pp. 1-37, 2008.

[20] M. M. Shoukri and A. Donner, "Bivariate modeling of interobserver agreement coefficients," Statistics in Medicine, vol. 28, no. 3, pp. 430-440, Feb. 2009.

[21] U. Engelke, A. Maeder, and H.-J. Zepernick, "Analysing interobserver saliency variations in task-free viewing of natural images," in Proc. of IEEE Int. Conf. on Image Processing, Sept. 2010. 